\title{
Consistent Set Theories With Universal Set
}

On occasion of the 1971 Berkeley symposium celebrating Alfred Tarski's achievements in logic and algebra Alonzo Church, who otherwise did not work much in set theory, presented a new system of set theory (Church 1974).

Church saw the two basic assumptions of post-naïve set theories in a restriction of comprehension to a form of separation (as in ZFC) and in a limitation of size (as in NBG). Similar to the criticism levelled against Limitation of Size in chapter II above Church regarded Limitation of Size as ad hoc (against the antinomies) and 'never well supported' as it proclaims a stopping point of further structures although classes are introduced (in NBG and MK) as collections, which can be quantified over. Church's set theory - let us call it "CST" here - follows ZFC in its idea of separation, but allows for collections that are 'large' in a way that even the larger transfinite sets of ZFC are not. CST does not introduce classes, but introduces a distinction within the area of sets. It allows even for $U=\{x \mid$ $\mathrm{x}=\mathrm{x}\}$.

CST distinguishes between 'low sets', which have a 1:1-relation to a wellfounded set, 'high sets', which are (absolute) complements of low sets and 'intermediate' sets which are neither. These labels pertain to the cardinality of sets. High sets are in 1:1-correspondence to the universal set $U$, low sets never. Because of the CST version of the Axiom of Choice for a set $x$ which is not low every ordinal has a 1:1-relation to some subset of $x$.

So the universe of CST may consist only of sets, but not all are wellfounded. Obviously $U \in U$. $U$ is the complement of $\varnothing$, so $U$ is the paradigmatic high set. $\varnothing$ is well-founded, and, of course, $\varnothing \in \mathrm{U}$. The constant predicate " $\mathrm{wf}(\mathrm{)})$ " expresses the property of BEING WELL-FOUNDED, defined in the usual sense (using some order relation " $<$ "):

$$
\begin{aligned}
& (\forall \mathrm{x})(\mathrm{wf}(\mathrm{x}) \equiv \mathrm{x}=\varnothing \vee \\
& (\forall \mathrm{y})(\mathrm{y} \subseteq \mathrm{x} \supset(\mathrm{y} \neq \varnothing \supset(\exists \mathrm{z})(\mathrm{z} \in \mathrm{y} \wedge(\forall \mathrm{w})(\mathrm{w} \in \mathrm{y} \supset \mathrm{z}<\mathrm{w}))))) .
\end{aligned}
$$

CST can be phrased as a second order system, quantifying over single- or two-argument open formula $\varphi$. One could understand this second order quantification as using classes, but only given a full-blown second order 
semantics [cf. Chap. II]. One could use schemata instead (i.e. having only free variables for open formula), as in $\mathbf{Z F}$. We follow the first option here and make CST a second order theory. ${ }^{36}$

The CST axioms are:

- Extensionality, Pair Set, Sum Set, Infinity as in $\mathbf{Z}$

- Choice: $(\forall \mathrm{R})((\forall \mathrm{x})(\exists \mathrm{y}) \mathrm{R}(\mathrm{x}, \mathrm{y}) \supset(\exists \mathrm{f})(\forall \mathrm{x}) \mathrm{R}(\mathrm{x}, \mathrm{f}(\mathrm{y})))$

- Product Set: $(\forall \mathrm{y}, \mathrm{z})(\mathrm{y} \in \mathrm{z} \supset(\exists \mathrm{u})(\forall \mathrm{x})(\mathrm{x} \in \mathrm{u} \equiv \mathrm{y} \in \mathrm{z} \supset \mathrm{x} \in \mathrm{y}))$

- Separation, Powerset, Replacement restricted to a condition " $\mathrm{wf}(\mathrm{x})$ ",

e.g. Axiom of Powerset: $(\forall x)(w f(x) \supset(\exists y)(\forall z)(z \in y \equiv z \subseteq x))$,

Axiom of Separation: $\quad(\forall \mathrm{x}, \mathrm{F})(\mathrm{wf}(\mathrm{x}) \supset(\exists \mathrm{y})(\forall \mathrm{z})(\mathrm{z} \in \mathrm{y} \equiv \mathrm{z} \in \mathrm{x} \wedge \mathrm{F}(\mathrm{z}))$

[where " $y$ " is not free in " $F$ "]

The Axiom of Product Set allows having a substitute for separation in high sets. What is missing is the Axiom of Foundation of $\mathbf{Z F}$.

These axioms of CST are strong enough to yield $\mathbf{Z F}$. Dropping the non well-founded sets one gains a $\mathbf{Z F}$ universe. The two theories are equiconsistent (cf. Church 1974, §5). And without violating this relative consistency CST can be extended by axioms which go beyond ZFC. These are: Strong Choice (that U can be well-ordered), Cardinality Axioms (that there are cardinal numbers in the sense of Frege and Russell for all wellfounded sets) and especially the Axiom of Complements:

$$
(\forall \mathrm{x})(\exists \mathrm{y})(\forall \mathrm{z})(\mathrm{z} \in \mathrm{y} \equiv \mathrm{z} \notin \mathrm{x}) \text {. }
$$

The existence of absolute complements and the existence of U make CST a more natural set theory than $\mathbf{Z F C}$, one may argue.

The argument in ZFC from the Axiom of Separation to the non-existence of $U$ and Cantor's Theorem pose no problem for $U$ and $\wp(U)$ for the same reason: the Axiom of Separation and the Axiom of Powerset are restricted to well-founded sets.

The argument to the non-existence of $\mathrm{U}$ [cf. Chap. I] now establishes that $\mathrm{U}$ is not a well-founded set. We knew that before. As U is not well-founded we do not have $\wp(\mathrm{U})$ in the first place.

One might now argue: So, in CST as well, there are some collections which are there - in this case inter alia the collection of all subsets of $U$, which are obviously existent if $U$ is - but cannot be collected into a set; some collections which should exist, like $\wp(\mathrm{U})$, do not exist, because they

36 Remember that in a full blown second order setting the Axiom of Choice is not equivalent to the Well-Order Principle. In fact the Well-Order Principle is not a theorem of ZFC2 (cf. Shapiro 1991, pp.106-108). Neither the presence of the Axiom of Choice nor the presence of Foundation implies that all sets can be well-ordered in ZFC2. 
are too large, just as in NBG set building operations cannot be applied to classes. Like NBG and MK tell us not much about classes, CST uses 'large' sets, but large sets cannot do much, since they are not subject to Separation or other set building principles.

This criticism, however, should be kept apart from a similar criticism levelled against NBG. In fact, all subsets of $U$ are collected into a set $-\mathrm{U}$ itself. What cannot be done is separating a set $\wp(\mathrm{U})$ from $U$. The same applies to the ordinals: $\mathrm{U}$ is $a$ set in which all ordinals are collected, but on pains of re-introducing the Burali-Forti antinomy - we cannot separate a set which collects only the ordinals. So does CST make some progress in comparison to NBG? On the one hand CST can avoid using the second ontological category of classes, with all its problems. On the other hand NBG can collect just the ordinals into their own proper collection, albeit a class. NBG cannot introduce $\wp(\mathrm{V})$, as CST cannot introduce $\wp(\mathrm{U})$.

It is true that although the principal idea behind CST was rejecting Limitation of Size, CST exhibits some shadow of Limitation of Size: All high sets are by definition of the same size as U, just as in NBG all classes are of the same size as the class of sets.

The widest known set theory with a universal set $U$ - for which we have $\mathrm{U} \in \mathrm{U}$ - is Quine's NF (from his paper "New Foundations of Mathematics", 1937). NF is Quine's set theory with a universal set that is not just virtual is NFU.

NF works by Extensionality and a Comprehension Scheme that is not as restricted as in $\mathbf{Z F C}$.

Quine's NF Comprehension Scheme uses the idea of stratified formula (similar to the simple theory of types):

$$
(\exists \mathrm{y})(\forall \mathrm{x})(\mathrm{x} \in \mathrm{y} \equiv \varphi(\mathrm{x}))
$$

where "y" is not free in $\varphi$ and $\varphi$ is stratified. A formula is stratified if the set on the right hand side of " $\in$ " is of a higher level than that on the left, and its definition does not include that on the left. A test for stratification consists in level assignments for sets or in trying a translation into the simple theory of types. The language of NF itself is not typed, thus avoiding duplication of structurally identical sets at different levels, but NF uses the stratification test to avoid the antinomies. NF is equiconsistent with the simple theory of types supplemented with the full ambiguity scheme, which asserts that a formula $\varphi$ is equivalent to formulas $\varphi^{1}, \varphi^{2} \ldots$ structurally similar to $\varphi$ with type levels uniformly raised by $1,2 \ldots$ 
NF has the power to introduce Pairing, (Absolute) Complement, Powerset, and Union as instances of Comprehension.

NF allows $U=\{x \mid x=x\}$, since " $x=x$ " is a stratified formula. $\{x \mid x \in y\}$ can be stratified, defining the so-called 'essence' of an object (the collection of all its properties). " $|\mathrm{x}|=|\{\varnothing,\{\varnothing\}\}| "$ is stratified as well, so natural numbers (in this case: 2) can be understood in Frege's way. Stratification excludes, however, the usual definition of an infinite set (as in the Axiom of Infinity in $\mathbf{Z}$ ). One meets in proofs and constructions in ZFC many unstratified set definitions. NF has to forsake these sets or has to introduce workarounds. Cardinal numbers and ordinal numbers come apart (cardinal numbers are not special ordinal numbers as in ZFC).

Non-stratified formula can be used in NF (this is different to Type Theory), but they cannot be used to define sets. Since non-stratified formulas can be used in NF one does not need a universal set or an empty set for every level (as in Russell's Type Theory) to have well-formed formula.

The antinomies - especially the (original) Russell Set - are avoided, since the corresponding open formulas in the Comprehension Scheme are not stratified. NF itself is not known to be consistent. NF with the Axiom of Counting, which says that a cardinal number is equal in cardinality to its singleton image, can prove the consistency of $\mathbf{Z}$. No relative consistency proofs to $\mathbf{Z F}$ are available. Note that instances of Replacement are not stratified. Some subsystems of NF have been shown to be consistent (cf. Forster 1992, Holmes 1999).

$\mathrm{U} \in \mathrm{U}$ means that Cantor's Theorem does not hold (in general) in NF; but the set of unit sets of its elements is smaller in cardinality than $U$ itself! The usual proof of Cantor's Theorem defines a set $\mathrm{y} \in \wp(\mathrm{x})$ relative to a supposed bijection $\mathrm{f}$ between $x$ and $\wp(\mathrm{x})$ as $\mathrm{y}=\{\mathrm{z} \mid \mathrm{z} \in \mathrm{x} \wedge \mathrm{z} \notin \mathrm{f}(\mathrm{z})\}$, which is not stratified. In NF one can define, however, $y=\{z \mid z \in x \wedge z \notin f(\{z\})\}$, which is stratified with $f$ being a supposed bijection between the set of singletons of $\mathrm{z} \in \mathrm{x}$ and $\wp(\mathrm{x})$. By the usual indirect argument one sees that there is no bijection between $\{\mathrm{z} \mid \mathrm{z}=\{\mathrm{w}\} \wedge \mathrm{w} \in \mathrm{x}\}$ and $\wp(\mathrm{x})$, which means $|\wp(\mathrm{x})|>|\{\mathrm{z} \mid \mathrm{z}=\{\mathrm{w}\} \wedge \mathrm{w} \in \mathrm{x}\}|=\left|\wp_{1}(\mathrm{x})\right|$. As $\wp(\mathrm{U}) \subseteq \mathrm{U}$ we have $|\mathrm{U}| \geq|\wp(\mathrm{U})|$ and thus with the previous inequality we know that the cardinality of the set of singletons of elements of $\mathrm{U}$ is smaller than $|\mathrm{U}|$ ! $|\mathrm{U}|>\left|\wp_{1}(\mathrm{x})\right|$. That could mean that not all elements of $U$ possess a singleton: although we have the set of all singletons in NF, as $\{\mathrm{x} \mid(\exists \mathrm{y})(\mathrm{x}=\{\mathrm{y}\})\}$ is stratified, not all objects seem to have a singleton. This cannot be the solution. Comprehension provides a singleton for any object $z$ : " $\mathrm{x} \in \mathrm{y} \equiv \mathrm{x}=\mathrm{z}$ " can be stratified. Contradiction is avoided finally by the non-existence of the function which maps any object to its singleton. This function does not exist even though every object has its singleton! 
NF has other highly controversial features like the existence of infinite descending chains of cardinals, what conflicts with the Axiom of Choice, which thus doesn't hold in NF, which again implies - even in the absence of an Axiom of Infinity - that the universe of NF has to be infinite, since all finite sets can be well-ordered. The universe of NF, supposedly U, cannot be well-ordered then. NF might be consistent, though, with the claim that all well-founded sets can be well-ordered. Some functions (like the successor function) are not part of the universe - so where are they?

One can extend NF by introducing classes. In fact if one denies in NF Rosser's Axiom of Counting [see above] one can prove the existence of non-set collections which are finite! Indeed a 'strange landscape' (Forster 1992, pp.29-32).

If one restricts the set building axioms to sets and uses unrestricted class comprehension one arrives at Quine's system ML (cf. Quine 1963, §§4042). The problems the system NF has with unstratified induction and its incompatibility with the Axiom of Choice are resolved then. There is a class of all sets, $\cup U$, and Cantor's Theorem does not apply to it, as it is no set (i.e. has no powerset at all). Apart from resolving these problems classes play no constructive role in ML. Obviously we find ourselves in a system very similar to MK, and corresponding criticism applies here [cf. Chap. II].

$*$

A version of NF that tries to avoid many of the peculiarities of NF is NFU (NF with urelements), developed by Randall Holmes (2005). NFU is built from NF by adding urelements and restricting extensionality to non-empty sets, introducing $\varnothing$ by an axiom. One may think of it as a subsystem of NF that allows only such models which contain the urelements.

NFU is consistent! It is consistent with the Axiom of Choice!

NFU is an extremely strong set theory. It can provide models for ZFCU by having very large cardinals (strongly inaccessible cardinals).

By working with a longer (finite) list of simple axioms (like Extensionality, Complements, Unions, Singletons, Cartesian Products, Converses, Domains, Projections, Singleton Image of a Set, Choice (!), Infinity...) Stratified Comprehension can be proven as a theorem!

The universal set is provided by its own axiom:

(U) $\quad\{\mathrm{x} \mid \mathrm{x}=\mathrm{x}\}$ exists.

U contains all sets as elements. All sets can be well-ordered, which is equivalent to the Axiom of Choice. So U can be well-ordered, in contrast to 
$\mathrm{U}$ in NF. By the existence of $\mathrm{U}$, the Axiom of Complements and Stratified Comprehension absolute complements exist.

NFU also has its peculiarities: urelements, atoms and ordered pairs (!) are taken as primitive. One wonders what is supposed to be 'in' the ordered pair $<\mathrm{x}, \mathrm{y}>$ if not $x$ and $y$. And the NFU Axiom of Ordered Pairs contains the standard identity condition on ordered pairs without saying that an ordered pair is a set. The Axiom of Pairing of $\mathbf{Z}$ looks more natural.

More problematically: $[\in],\{<\mathrm{x}, \mathrm{y}>\mid \mathrm{x} \in \mathrm{y}\}$, does not exist, the existence of $[\in]$ leading to antinomies. ${ }^{37}$ Whereas we thus have a set $U$, which may stand in as the extension of the predicate "set", we have no extension for the membership relation, although $[\in]$ looks as natural as U. Interestingly $[\subseteq]=\{<\mathrm{x}, \mathrm{y}>\mid \mathrm{x} \subseteq \mathrm{y}\}$ exists. So in many cases " $\{\mathrm{x}\} \subseteq \mathrm{y}$ " may substitute for " $\mathrm{x} \in \mathrm{y}$ ".

The non-existence of $[\in]$, which, of course, entails $[\in] \notin \mathrm{U}$, entails that the membership relation is not modelled by $U$ ( $U$ has no element corresponding to it), so that $U$ cannot be a model for NFU itself. So as with the standard set theories $\mathbf{Z F}$, ZFC, although working with a universal set, NFU has to look outside of itself for models. If the universal set is really universal, where should that outside be? We seem to be back to larger cardinals or similar collection like entities or some hierarchy [cf. Chap. I \& II].

Not every supposed set exists, e.g. the set of all Cantorian ordinals. This is not better than in $\mathbf{Z F}$. Some collections (like U) which do not exist in ZFC can exist in NFU, but as NFU does not distinguish sets from classes, some collections (like that of Cantorian ordinals) which exist in NBG do not exist in NFU. NFU can be viewed as trying to capture some middle ground between the other systems. This yields its own peculiarities.

Especially problematic is that some version of Cantor's Theorem is provable. The large sets (like the set of ordinals or U) in NFU have the strange property of not being equinumerous to their singleton images! How can that be? Although every object has a singleton (by the Axiom of Singletons), just as in NF the function giving the singleton to every object does not exist. ${ }^{38}$

$\left|\wp_{1}(\mathrm{U})\right|<|\mathrm{U}|$ seems to contradict the Axiom of Singletons, a provable contradiction only being avoided by the non-existence of a general

37 Proof (Outline). If $[\in]$ exists, so does its complement $-[\in]$ by the Axiom of Complements. $[=]=\{<\mathrm{x}, \mathrm{y}>\mid \mathrm{x}=\mathrm{y}\}$ exists, since " $\mathrm{x}=\mathrm{y}$ " is stratified. Then the cut of $[=]$ with $-[\in]$ exists, and this cut is a cousin of the Russell Set: $\{<x, y>\mid x=y \wedge x \notin y\}$. 38 Proof (Outline). If the function $f: \mathrm{U} \rightarrow \wp_{1}(\mathrm{U})$ existed, extensionality of the singleton would yield a function $f^{-1}$, so $|\mathrm{U}| \leq\left|\wp_{1}(\mathrm{U})\right|$, which contradicts the Cantorian argument to $\left|\wp_{1}(\mathrm{U})\right|<|\mathrm{U}|$ (given above with respect to $\mathbf{N F}$ ).

Holmes (2005, pp. 109-110) provides another proof which relates to stratification. 
singleton function (cf. Quine 1963, p.293). We have - so to say, in analogy to Separation restricting Comprehension in $\mathbf{Z}$ - restricted singleton construction!

So a corollary of the non-existence of the (general) singleton function is that the cardinality of the set of singletons of members of $U,\left|\wp_{1}(\mathrm{U})\right|$, is less than the cardinality of $U$. This leads in NFU to the distinction between 'Cantorian sets' $x$ with $|\mathrm{x}|=\left|\wp_{1}(\mathrm{x})\right|$ and 'non-Cantorian sets', which resembles other limitation of size distinctions.

These cardinality issues lead to Specker's Theorem (cf. Holmes 2005, pp.132-34):

$$
|\wp(\mathrm{U})|<|\mathrm{U}|
$$

which is read as proof that there are (many, many) urelements/atoms besides sets in U. Atoms, which have no members, are not elements of $\wp(\mathrm{U})$, which contains all subsets of $\mathrm{U}$, but not non-sets like atoms. If there are no atoms one expects for a system with a universal set $U$ that one has $|\wp(\mathrm{U})|=|\mathrm{U}|$. In fact most objects in $\mathrm{U}$ in NFU then have to be atoms or pairs (i.e. ordered pairs not reducible to sets).

Unlike some version of NF in (Quine 1963) atoms are not identified with their singletons in NFU. As mentioned, ordered pairs are also objects in their own right besides sets. Ordered pairs can be taken as atoms in NFU as no claim with respect to composition - only with respect to their identity condition - was made. Specker's Theorem mirrors this conception.

Again:

Specker's Theorem for NFU asserts that most entities in U are not subsets of $\mathrm{U}$, which means most of the universe has to consist of urelements!

So: in all known models of NFU $|\mathrm{U}|>|\wp(\mathrm{U})|$ ! All relations are subsets of $\mathrm{U} \times \mathrm{U}$, and all functions $\mathrm{f}: \mathrm{U} \rightarrow \mathrm{U}$ should, if they are allowed to exist, themselves be sets of ordered pairs, thus be elements of $U$, thus be available as their own arguments, thus sometimes be forbidden to exist $a s$ sets at all in a consistent setting (e.g. a function of negative selfapplication). NFU itself can have models only in a realm which possesses properties quite different from what we expect of sets. NFU, so, deals not just with sets. In fact the non-sets vastly outnumber the sets in any model of NFU. Ideally the non-sets contain just the urelements, but by the argument above concerning the Axiom of Singletons we should expect there to be a collection containing the ordered pair of any $x$ and its singleton, but this collection cannot be a set in NFU. A crucial question is whether NFU can at least recapture ordinary sets - ZFC-like entities - as a sub-domain. Even though this is possible, however, we regain simply $\mathbf{Z F C}$ as a sub-universe - and are none the wiser with respect to our universality problem, as U, 
because of its behaviour in NFU, cannot be part of that recaptured realm. $U$ is not 'Cantorian'. Even the ordinals of NFU cannot be well-ordered in a set model of NFU! Holmes does not introduce proper classes into NFU, but admits their existence, supposedly objects of a broader theory (cf. Holmes 2005, p.50). NFU is not truly universal, as well.

NFU, thus, may provide a lot of machinery to do ordinary mathematics, the gain with respect to our set theoretic intuitions brought by having a universal set $\mathrm{U}$, however, seems to be more than lost by the consequences of Specker's Theorem and the absence of $[\in]$. 